\title{
Fogitare
}

\section{PRÁTICAS DE CUIDADOS DO ENFERMEIRO NA ATENÇÃO PRIMÁRIA À SAÚDE: GESTÃO DO CUIDADO DA PELE DO IDOSO*}

\author{
Francisco Reis Tristão ${ }^{1}$, Juliana Balbinot Reis Girondi ${ }^{2}$, Karina Silveira de Almeida \\ Hammerschmidt ${ }^{3}$, Katheri Maris Zamprogna ${ }^{4}$, Cilene Fernandes Soares ${ }^{5}$, Scheila Monteiro \\ Evaristo ${ }^{6}$, Amanda de Souza Vieira ${ }^{7}$
}

\section{RESUMO}

Objetivo: identificar práticas de cuidado empregadas pelos Enfermeiros da Estratégia Saúde da Família para prevenção, diagnóstico de enfermagem e tratamento de lesão por fricção e lesão por pressão em idosos na comunidade.

Método: estudo qualitativo descritivo com 25 enfermeiros da Estratégia Saúde da Família, lotados no âmbito da Secretaria Municipal de São José/Santa Catarina. Para coleta de dados, aplicou-se questionário semiestruturado entre março e maio de 2018. Os dados foram submetidos à Análise Temática de Conteúdo.

Resultados: emergiram quatro categorias distintas relacionadas às práticas do enfermeiro para cuidado da pele do idoso: Ferramentas para avaliação clínica da pele do idoso; Avaliação do risco para as lesões em idosos; Estadiamento das lesões; e Tratamento das lesões em idosos.

Conclusão: observou-se a necessidade de maior investimento institucional em ações de educação permanente aos profissionais de Enfermagem, para que sejam efetivadas boas práticas de cuidado na prevenção, estadiamento e manejo das lesões estudadas.

DESCRITORES: Enfermagem em Saúde Comunitária; Enfermagem Geriátrica; Cuidados de Enfermagem; Pele; Ferimentos e Lesões.

\footnotetext{
*Artigo extraído da dissertação de mestrado "Skin help: mínimo produto viável para aplicativo de apoio à decisão na prevenção, diagnóstico de enfermagem e tratamento de lesão por pressão e fricção em idosos." Universidade Federal de Santa Catarina, 2018.
}

COMO REFERENCIAR ESTE ARTIGO: Tristão FR, Girondi JBR, Hammerschmid KS de A, Zamprogna KM, Soares $\mathrm{CF}$, Evaristo $\mathrm{SM}$, et al. Práticas de cuidados do enfermeiro na atenção primária à saúde: gestão do cuidado da pele do idoso. Cogitare enferm. [Internet]. 2020 [acesso em "colocar data de acesso, dia, mês abreviado e ano"]; 25. Disponível em: http://dx.doi.org/10.5380/ce.v25i0.65223.

Este obra está licenciado com uma Licença Creative Commons Atribuição 4.0 Internacional.

${ }^{1}$ Enfermeiro. Mestre em Gestão do Cuidado em Enfermagem. Gerência de Distrito Sanitário da Diretoria de Atenção Primária à Saúde de São José. Florianópolis, SC, Brasil. 9

${ }^{2}$ Enfermeira. Doutora em Enfermagem. Docente de Enfermagem e de Pós-Graduação em Gestão do Cuidado em Enfermagem da Universidade Federal de Santa Catarina. Florianópolis, SC, Brasil. 0

${ }^{3}$ Enfermeira. Doutora em Enfermagem. Docente de Enfermagem da Universidade Federal de Santa Catarina. Florianópolis, Santa Catarina, Brasil. (2)

${ }^{4}$ Enfermeira. Mestre em Enfermagem. Gerência de Distrito Sanitário da Diretoria de Atenção Primária à Saúde de São José. Florianópolis, SC, Brasil. (1)

${ }^{5}$ Enfermeira. Mestre em Enfermagem. Enfermeira do Hospital Universidade Polydoro Ernani de São Thiago. Florianópolis, SC, Brasil.

${ }^{6}$ Enfermeira. Especialista em Atenção à Saúde da Pessoa Idosa. Gerência de Unidade Básica de Saúde, Secretaria Municipal de Saúde de São José. Florianópolis, SC, Brasil. (2)

${ }^{7}$ Discente de Enfermagem. Universidade Federal de Santa Catarina. Florianópolis, SC, Brasil. ( 


\title{
NURSING CARE PRACTICES IN PRIMARY HEALTH CARE: ELDERLY PEOPLE'S SKIN CARE MANAGEMENT
}

\begin{abstract}
Objective: to identify care practices used by nurses who work at the Family Health Strategy program to prevent and treat friction and pressure injuries in elderly people living in the community and perform the corresponding nursing diagnosis.

Methods: qualitative and descriptive study carried out with 25 Family Health Strategy nurses, linked to the Municipal Health Secretariat of São José, Santa Catarina, Brazil. A semistructured questionnaire was applied between March and May 2018 to collect data, which were submitted to thematic content analysis.

Results: four different categories related to nursing practices for elderly people's skin care emerged: tools for clinical evaluation of elderly people's skin; evaluation of risk of injuries in elderly people; injury staging; and treatment of the injuries in elderly people.

Conclusion: the need for higher institutional investment in continuing education actions oriented toward nursing professionals was observed, so good care practices can be implemented in preventing, staging, and handling the injuries under discussion.
\end{abstract}

DESCRIPTORS: Community Health Nursing; Geriatric Nursing; Nursing Care; Skin; Wounds and Injuries.

\section{PRÁCTICAS DE ENFERMERÍA EN ATENCIÓN PRIMARIA DE SALUD: GESTIÓN DEL CUIDADO DERMICO DEL ANCIANO}

\begin{abstract}
RESUMEN
Objetivo: Identificar prácticas de atención utilizados por enfermeros de Estrategia Salud de la Familia en prevención, diagnóstico de enfermería y tratamiento de lesión por fricción y por presión en ancianos.

Método: Estudio cualitativo, descriptivo, con 25 enfermeros de Estrategia Salud de la Familia, actuantes en el ámbito de la Secretaría Municipal de São José/Santa Catarina. Datos recolectados mediante cuestionario semiestructurado, entre marzo y mayo de 2018, posteriormente sometidos a análisis temático de contenido.

Resultados: Surgieron cuatro categorías en relación a las prácticas del enfermero para cuidar la piel del anciano: Herramientas de evaluación clínica de la piel del anciano; Evaluación del riesgo de lesiones en ancianos; Estadificación de las lesiones; y Tratamiento de las lesiones en ancianos.

Conclusión: Se observó necesidad de mayor inversión institucional en capacitación continuada a profesionales de enfermería, para que sean aplicadas las buenas prácticas de atención en prevención, estadificación y manejo de las lesiones estudiadas.
\end{abstract}

DESCRIPTORES: Enfermería en Salud Comunitaria; Enfermería Geriátrica; Atención de Enfermería; Piel; Heridas y Traumatismos. 
O aumento da população idosa é considerado evento global. Assim, o serviço de saúde precisa estar preparado para atender à especificidade das demandas inerentes a este seguimento etário. No idoso, são comuns alterações na pele como o ressecamento cutâneo, descamação, afinamento, redução da elasticidade, dentre outras, consideradas inerentes ao envelhecimento. Não obstante, associadas a estas condições, e sob influência de fatores externos (pressão, fricção, cisalhamento), esta clientela apresenta maior chance de desenvolver lesões de pele, dentre elas, a Lesão por Fricção (LF) e Lesão por Pressão $(\mathrm{LP})^{(1-2)}$.

A LF ocorre quando há ruptura da pele, decorrente de trauma (fricção, contusão, cisalhamento) capaz de ocasionar separação da composição tecidual (epiderme, derme, ou ambas). A LP configura dano tecidual, superficial ou profundo, decorrente de pressão intensa ou prolongada, e/ou cisalhamento, que em grande parte ocorre sobre áreas de proeminências ósseas ou artefatos (médicos ou não) ${ }^{(1,3)}$.

Neste contexto, considera-se que a prática assistencial do Enfermeiro deve se fundamentar na avaliação criteriosa da pele do idoso, para que, por meio de exame físico, possam ser elucidados diagnósticos de enfermagem e subsequentes intervenções, capazes de tratar ou prevenir complicações inerentes à alteração ou perda da integridade cutânea do idoso ${ }^{(4-5)}$.

Vale ressaltar que na Atenção Primária à Saúde (APS), por meio da Estratégia Saúde da Família (ESF), a atuação do Enfermeiro está centrada na abordagem participativa e alicerçada na integralidade do cuidado, o que permite infindas possibilidades para - enfrentamento dos agravos sofridos pela população idosa, dentre eles, aqueles relacionados à pele. Logo, dada a complexidade do cuidado, a APS constitui cenário essencial ao Enfermeiro para a fundamentação de práticas preventivas, uma vez que sua inserção na comunidade possibilita maior reconhecimento das demandas em saúde deste grupo etário, o que permite a este profissional identificar e programar ações que visem manter a integridade da pele do idoso ${ }^{(6-7)}$.

Frente ao exposto, o objetivo deste estudo é identificar práticas de cuidado empregadas pelos Enfermeiros da Estratégia Saúde da Família para prevenção, diagnóstico de enfermagem e tratamento de lesão por fricção e lesão por pressão em idosos na comunidade.

\section{MÉTODO}

Trata-se de estudo qualitativo, descritivo. A investigação foi realizada junto aos Enfermeiros das equipes de ESF, do município de São José-SC, que possui em sua Rede Assistencial 23 Unidades Básicas de Saúde, equivalente a 44 equipes de ESF, e cuja população é estimada em 242.927 habitantes, sendo que 19.843 possuem idade igual ou superior a 60 anos $^{(8)}$.

Para compor a amostra, foram convidados a participar Enfermeiros da ESF, lotados no âmbito da instituição supracitada, cuja população correspondia a 44 profissionais, conforme Departamento de Informática do SUS (DATASUS) ${ }^{(9)}$. Para tanto, enquanto critérios de inclusão foram selecionados servidores, efetivos ou contratados, com atuação superior a três meses no município e que compunham alguma equipe de ESF. Foram excluídos aqueles que estavam em período de férias ou licença gestação/tratamento de saúde/ prêmio, ou em qualquer outro tipo de afastamento.

Os participantes foram convidados mediante reunião de Comissão de Enfermeiros, onde se explanou os propósitos do estudo. Posteriormente, foi realizado contato individual 
para agendamento das entrevistas, tendo 25 enfermeiros aptos conforme critérios de inclusão, em que foi aplicado questionário semiestruturado, e realizada coleta dos dados de março a maio de 2018, até que fosse observada saturação das informações pelo pesquisador ${ }^{(10)}$. As coletas foram realizadas nas Unidades Básicas de Saúde e/ou Secretaria Municipal de Saúde, com a duração das entrevistas de em média 25 minutos.

Os dados provenientes das entrevistas foram transcritos por meio da plataforma Google Forms $囚$, a qual permitiu análise descritiva das informações coletadas. Os dados foram submetidos à Análise Temática de Conteúdo(11).

O estudo foi aprovado pelo Comitê de Ética em Pesquisa com Seres Humanos da Universidade Federal de Santa Catarina, por meio do parecer consubstanciado n².697.902.

\section{RESULTADOS}

Participaram do estudo 25 Enfermeiros, sendo 22 (88\%) do sexo feminino. A idade dos participantes variou de 26 a 56 anos, com tempo de atuação na APS de inferior a seis meses a 16 anos, sendo $11(44 \%)$ atuantes entre um e cinco anos no local. Quanto à escolaridade, $13(52 \%)$ possuíam nível pós-graduação lato sensu, $11(44 \%)$ nível superior completo, e um participante possuía mestrado.

Dos resultados, emergiram quatro categorias: Ferramentas de avaliação clínica; Avaliação de risco de lesão por fricção e pressão; Instrumentos para o diagnóstico de enfermagem; e Tratamento, destacadas abaixo.

\section{Categoria 1 - Ferramentas para avaliação clínica da pele do idoso: práticas do Enfermeiro na Atenção Primária à Saúde}

Observou-se divergência entre as falas e ausência de padronização de ferramentas na instituição à qual os enfermeiros eram vinculados. Os profissionais mencionaram utilizar as seguintes aplicações:

Protocolo para prevenção de úlcera do Ministério da Saúde. (E2)

Escala de Braden (avaliação grau de percepção sensorial, umidade, atividade física, nutrição, fricção e cisalhamento). (E5)

Para a avaliação de pele, estou utilizando o RYB e o Measure. (E11)

Utilizo a escala de Braden. Também temos por costume utilizar a escala de Norton. (E17)

Acrescenta-se que uma das entrevistas ressaltou a relevância de avaliação clínica da pele, diante da presença de LF:

[...] Seja na atenção básica ou âmbito hospitalar, quando presenciamos as lesões por fricção, principalmente em idosos [...] utilizo a padronização STAR, que me ajuda a classificar a categoria/estadiamento da lesão, por exemplo. (E25)

[...] Pode ser utilizada a escala de Braden [...] o risco para o desenvolvimento de lesão por pressão também é baseado no estilo de vida, no uso ou não de tabagismo, dentre outros aspectos. (E25)

\section{Categoria 2 - Avaliação do risco para lesão por fricção e lesão por pressão em idosos: ações e cuidados de enfermagem para prevenção na Atenção Primária à Saúde}

No intuito de compreender de que forma é avaliado o risco para LF e LP em idosos 
pelos Enfermeiros na APS, observou-se que a aplicação de ferramentas validadas para este fim é pouco explorada.

Avalio quanto à falta de mudança de decúbito, condições inadequadas do colchão, higiene corporal inadequada, umidade da pele, nutrição. (E1)

Na verdade, faço avaliação conforme conhecimentos teóricos. (E4)

Não utilizo escalas ou protocolos. (E5)

Levo em conta as condições externas e internas que contribuem para o aparecimento dessas lesões, embora as escalas serem consideradas instrumentos confiáveis. Não consigo adequar à realidade. (E10)

Avaliação empírica, de acordo com a minha experiência [...]. (E12)

[...] através da anamnese e dos fatores de risco para desenvolvimento de lesões. (E16)

[...] avaliação da pele, avaliação do estado nutricional, hidratação, distúrbios imunológicos, deficiências, situação de moradia, situação econômica para verificarmos melhor conduta a ser tomada pela equipe. (E17)

Avalio através da coloração da pele, umidade desta, capacidade do paciente reagir significativamente à pressão relacionada do desconforto, se paciente é acamado ou não, quanto à mobilidade do paciente, nutrição do paciente. (E20)

Quando empregadas escalas, denota-se majoritariamente o uso de ferramentas para estratificação do risco de LP:

[...] levo em conta o tipo de pele, roupa que faz uso, e por último faço uso da escala de Braden para lesão por pressão. (E19)

Em virtude de questões de processo de trabalho, alguns Enfermeiros relatam não utilizar ferramentas para avaliação de risco:

Não utilizo no dia-a-dia devido à grande demanda na unidade e à falta de tempo. (E5)

Não, pois ainda estamos sem protocolo de curativo. (E15)

Não, visto a grande demanda na unidade e consequentemente pouco tempo disponível para avaliação de todos os idosos em visitas domiciliares. (E22)

No município não existe protocolo direcionado para esta faixa etária. (E13)

Os dados sugerem que a definição de plano de cuidados preventivos às LF e LP parece estar alicerçada ao fator de risco apresentado pelo idoso. Assim, observa-se tendência à proposição de cuidados relacionados à prevenção de LP e com menor frequência para LF, conforme os seguintes cuidados preventivos:

Mudança de decúbito, posicionamento no leito, hidratação, nutrição, cuidados com a pele, observar mudanças na pele. (E5)

Manter paciente seco e hidratado; pele higienizada; dieta e hidratação; reposicionamento; uso de colchão adequado, almofadas e coxins quando paciente sentado apoiar pés, deitado inclinar no máximo $30^{\circ}$ cabeceira; nos acamados uso de forro móvel, proteção calcâneos, manter membros inferiores e superiores protegidos. (E7)

Uso de colchão adequado (piramidal); mudança de decúbito; hidratação; alimentação adequada (vitaminas e proteínas); cobertura de acordo com aspecto da lesão. (E15)

Melhorar dieta, mudança de decúbito 2/2h, hidratação da pele, diminuição da fricção, tirar 
bordas e irregularidade no lençol e colchão, se possível usar colchão pneumático. (E18)

Para prevenção de LF, observou-se enquanto prática o uso de:

[...] sabonete $\mathrm{pH}$ balanceado; água morna para lavagem da fricção (lesão); redução do tempo de banho; aplicação de creme umectante no corpo, não nas lesões; dieta balanceada. (E15)

Além disso, acrescenta-se que, caso observado fator de risco relacionado à $\mathrm{LF}$, foram pontuadas enquanto práticas de cuidado para a prevenção:

[...] evitar uso de esponjas na pele ao tomar banho; evitar medicações e/ou produtos adesivos; aplicação de hidratantes; água do banho morna; manter unhas aparadas; aumentar ingesta hídrica. (E19)

\section{Categoria 3 - Instrumentos para o diagnóstico de enfermagem: aplicação de Sistemas de Classificação para lesão por fricção e lesão por pressão pelo Enfermeiro na Atenção Primária à Saúde}

Quando questionados sobre o uso dos sistemas de classificação ou de diagnóstico de enfermagem para LF e LP, os participantes do estudo demonstraram pouca familiaridade com a utilização de instrumentos:

Não utilizo, pois não existe [...]. (E12)

Classifico de acordo com a etiologia da lesão, mas não utilizo nenhum sistema. (E16)

Não utilizo sistemas, não tenho conhecimento sobre eles. (E23)

Também foi observada a aplicação de nomenclatura específica para estadiamento da LP no cenário de investigação. Ademais, evidenciou-se que, no contexto observado, o estadiamento da LF ainda é incipiente.

Classificação por estágio, conforme NPUAP. (E7)

National Pressure Ulcer Advisory Panel (NPUAP). As lesões são classificadas: Estágio 1; Estágio 2; Estágio 3; Estágio 4; Lesão por pressão tissular profunda; Lesão por pressão não estável; Lesão por pressão relacionados a dispositivos médicos; Lesão por pressão em membrana mucosa. (E10)

Em se tratando especificamente de LF, apenas um Enfermeiro pontuou:

No que tange a skin tear [...] utilizo a STAR para nomenclatura de estadiamento. (E25)

Acrescenta-se que outros profissionais mencionaram o uso de classificação para estadiamento da LP, porém não especificaram a origem do sistema empregado:

Utilizo por estadiamento a classificação de lesão por pressão em estágio 1, 2, 3, 4. (E10)

Quanto à lesão por pressão, a sua classificação ocorre em 4 estágios (1, 2, 3, 4), os quais são sim utilizados por mim e minha equipe de trabalho na ESF, para classificar a lesão por pressão. (E25)

\section{Categoria 4 - Tratamento e plano de cuidados de lesão por friç̧ão e lesão por pressão em idosos: práticas empregadas pelo Enfermeiro na Atenção Primária à Saúde}

Em relação ao tratamento, ratificou-se a aplicação de práticas voltadas aos aspectos gerais para o manejo das lesões de pele, e quando especificados, quase sempre eram relacionadas à LP: 
Conforme avaliação da úlcera para sinais de infecção. Se houver necrose, considerar desbridamento (encaminhar). Limpeza da úlcera e pele ao redor, em cada mudança de curativo. Nutrição da pele. Coberturas apropriadas para manutenção da ferida. Orientação quanto a medidas de prevenção. (E4)

Inspeção da pele; manter paciente seco e hidratado; pele higienizada; dieta e hidratação; reposicionamento; uso de colchão adequado, almofadas e coxins quando paciente sentado apoiar pés, deitado inclinar no máximo $30^{\circ}$ cabeceira; nos acamados uso de forro móvel, proteção calcâneos, manter membros inferiores e superiores protegidos. (E7)

Para LP uso de coxins para aliviar a pressão do local; uso de produtos que auxiliam na cicatrização; mudança de decúbito; uso de curativos, limpeza do local afetado e avaliação do leito da ferida. (E10)

Os cuidados referentes ao tratamento de LF foram pontuados por dois Enfermeiros.

Realizo um plano de cuidados para o tratamento da ferida, realização de curativos, se houver retalho em lesão por fricção, aproximação das bordas; retirada de curativo de forma atraumática; uso de produtos para ajudar na cicatrização. (E19)

Realizo intervenções e cuidados baseados no tipo de lesão, [...] não é viável a padronização ao cuidado de lesão por pressão e skin tear, uma vez que a depender do grau, a pele irá se apresentar de diferentes formas, exigindo tratamentos distintos [...] fazendo-se pertinente por vezes o uso de hidrogel, a depender do aspecto da lesão, bem como espumas, espuma de prata, alginato, dentre outros. (E25)

\section{DISCUSSÃO}

O perfil sociodemográfico da amostra foi caracterizado por profissionais do sexo feminino, remontando ao fato histórico da Enfermagem ser profissão majoritariamente composta por mulheres ${ }^{(12)}$.

Quanto ao conhecimento das ferramentas para as práticas destes profissionais no gerenciamento da LF e LP, os achados evidenciam ausência de padronização institucional, sugerindo a necessidade de fundamentar ações e cuidados de enfermagem em prática baseada em evidências, no intuito de garantir a assistência de qualidade ao idoso(13). Corroboram com essa questão estudiosos da área ${ }^{(14)}$ ao destacarem que a qualificação da prática do Enfermeiro na APS deve ser apoiada a partir da consolidação de protocolos assistenciais, os quais propiciam conhecimento científico, respaldando o profissional na tomada de decisão.

É relevante acrescentar que, independente da ausência (ou presença) dessas ferramentas, a avaliação da pele do idoso deve ocorrer em concomitância ao Processo de Enfermagem, iniciado por meio da anamnese ${ }^{(4)}$. Neste estudo, as ferramentas utilizadas pelos Enfermeiros, embora mencionadas com esta finalidade, não estavam atreladas especificamente à avaliação clínica da pele, mas à estratificação de risco, estadiamento ou caracterização de lesões, como é o caso, por exemplo, da Escala de Braden, da Classificação STAR e do Sistema RYB ${ }^{(15-17)}$.

Fatores como a sobrecarga de trabalho também parecem interferir na utilização destes recursos na prática clínica, sugerindo que adequações no quantitativo de Enfermeiros ao processo de trabalho seja discutido ${ }^{(14)}$.

Observa-se a estratificação de risco para LF e LP de maneira pouco sistematizada. Contudo, salienta-se que nas ocasiões em que o cuidado se deu através do uso de escala, observou-se congruência quanto ao emprego da Escala de Braden, indo ao encontro do estudo realizado que avaliou variáveis sociodemográficas, clínicas e aplicação das Escalas 
de Waterlow e Braden e percebeu que a de Braden mostrou-se como meio de triagem mais eficaz se comparado à de Waterlow ${ }^{(18)}$.

No que concerne à LF, constatou-se que, apesar de existirem ferramentas para classificação de risco, há desconhecimento por parte dos participantes deste estudo quanto a sua utilização. Possivelmente, este achado se deve ao fato desta ainda ser temática incipiente no Brasil, embora a literatura indique a realização de estudos estrangeiros na área desde a década de 1990(16).

Consoante, ainda que não tenha sido validada no Brasil, autores ${ }^{(19)}$ sugerem a aplicação da Skin Tear Risk Assessment Pathway, uma ferramenta elaborada por um consenso de especialistas que tem por finalidade estratificar os riscos para ocorrência de LF.

Em relação às práticas preventivas, observou-se que as ações relacionadas à LP corroboram com o descrito na literatura, como reposicionamento, cuidados em relação aos aspectos nutricionais, proteção de calcâneo e uso de colchão específico(20-21). Quanto ao reposicionamento, foi apontado como prática para prevenção de LP pelos participantes. Apesar de não mencionado pelos entrevistados, estudos acrescentam que se deve adotar posição de $30^{\circ}$, podendo-se ajustar a 45 graus, quando confirmada obesidade, bem como reposicionamento em tempo inferior a três horas ${ }^{(22-23)}$.

O uso de hidratantes também foi apontado como intervenção eficaz para a prevenção de LP. Conforme o National Pressure Ulcer Advisory Panel (NPUAP), o European Ulcer Advisory Panel (EPUAP) e a Pan Pacific Pressure Injury Alliance (PPPIA)(24), a utilização de emolientes para hidratação da pele deve ser considerada, uma vez que reduz risco de dano à pele.

Quando mencionados os cuidados relacionados à LF pelos participantes do presente estudo, observa-se que estes seguem em consonância com o sugerido pelo International Skin Tear Advisory Panel, com ressalva quanto a: aplicação de hidratantes ao menos duas vezes ao dia, adoção de produtos adesivos à base de silicone, uso de roupas com manga longa, atenção quanto a toque brusco na pele do idoso ${ }^{(1,6,25)}$.

Chama a atenção que a hidratação da pele e o uso de água morna durante o banho destacaram-se pelos entrevistados, sendo cuidados importantes, já que configuram parte das ações necessárias para manutenção da integridade da pele e consequente prevenção de $\operatorname{LF}^{(26)}$.

Em se tratando do diagnóstico das lesões estudadas, não foi observado o emprego de diagnósticos de enfermagem especificamente relacionado a LF e LP, contudo, observouse adoção de instrumentos que auxiliam sua elucidação.

No que concerne à LP, a literatura aponta que a NPUAP dispõe de sistema de classificação desde 1989, passando por sucessivas atualizações, sendo a mais recente publicada em $2016^{(3)}$. Atualmente, o referido sistema classifica a LP em estágios que variam do um ao quatro ${ }^{(3)}$. Alguns Enfermeiros relataram classificar a LP com base em estágios, sem pontuarem o sistema empregado. Possivelmente, estes profissionais referem-se ao proposto pela NPUAP(27), haja vista similaridade com a nomenclatura supramencionada.

Em relação à $L F$, embora considerada tema incipiente no Brasil, há registros na literatura de que a abordagem a estas lesões já vem sendo discutida desde 1990, quando proposto por Payne e Martin o sistema "Classification System for Skin Tears"(28).

Os achados demonstram que práticas de cuidado como controle da umidade, reposicionamento, angulação da posição, hidratação, suspensão do calcâneo, uso de coxins, dentre outros, são comumente aplicadas pelo Enfermeiro no contexto investigado. Cabe inferir que, embora não se exclua a necessidade de aplicá-las durante o tratamento, a literatura pontua estas práticas, enquanto intervenções relacionadas à prevenção da $\mathrm{LP}^{(23,27) \text {. }}$ 
Em relação à terapia tópica, mencionou-se a relevância do uso de coberturas para o tratamento, contudo, não foram citadas tecnologias específicas, com exceção do desbridamento, apontado para fins de necrose tecidual. Vale acrescentar que esta técnica tem sido apontada como possibilidade ao tratamento da LP, conforme descrito pelos autores ${ }^{(29)}$, porém, parece não ser eficaz quando aplicada isoladamente. Em se tratando dos cuidados das LF, apontado por discreta parcela dos participantes, os cuidados mencionados coadunam ao proposto pela literatura(30), que sugere a terapia tópica enquanto cobertura, desde que haja retalho de pele viável, passível de realinhamento.

No que diz respeito à retirada atraumática do adesivo, esta medida tem sido mais associada à prevenção de LF, do que ao tratamento da lesão. Porém, ao deparar-se com a necessidade deste uso, o Enfermeiro deve dar preferência a fitas de baixa capacidade adesiva ${ }^{(30)}$. Chama atenção o fato de uma das entrevistadas mencionar que a padronização do cuidado deve ser evitada, uma vez que a condução do tratamento está diretamente relacionada às características da lesão.

Em se tratando da classificação da lesão, o tratamento pode variar de coberturas à base de espuma de silicone, uso de alginato de cálcio, hidrofibra, hidrogel, prata iônica, azul de metileno, cola à base de 2 Occtil-cianoacrilato, dentre outras tecnologias ${ }^{(6,30)}$.

A investigação junto a uma parcela circunscrita da Enfermagem pode configurar uma limitação do presente estudo. Assim, sugere-se que futuras investigações sejam realizadas, em diferentes regionalidades, proporcionando comparação dos achados e evidenciando as práticas despendidas pelos profissionais, em macrocenário.

\section{CONCLUSÃO}

A ausência de protocolos institucionais para a condução do cuidado às lesões estudadas pareceu exercer influência nas ações despendidas pelos Enfermeiros, sendo comuns divergências quanto às práticas de cuidado exercidas, e aleatória, quando presente, a seleção de ferramentas para o gerenciamento do risco e manejo de ambas as lesões.

Dentre as lesões estudadas, as LP foram as que os Enfermeiros demonstraram deter maior conhecimento, especialmente quanto à estratificação de risco e práticas preventivas à ocorrência deste agravo. Certamente este achado é consequência dos estudos de longa data por pesquisadores brasileiros sobre a estratificação de risco para LP através da escala supracitada.

Observou-se pouca familiaridade dos Enfermeiros quanto à $L F$, possivelmente em virtude das discussões acerca desta temática no Brasil terem ganhado força aparentemente nos últimos anos. Evidencia-se a necessidade de investimento institucional em ações de educação permanente aos profissionais, bem como na construção de ferramentas locais para sistematização do cuidado, induzindo boas práticas à prevenção, estadiamento e manejo das lesões, haja visto seu impacto ao usuário e ao serviço de saúde.

Acredita-se que os resultados do presente estudo encorajarão a comunidade científica a refletir acerca do cuidado a estas lesões, vislumbrando a proposição e incorporação de novas tecnologias, as quais sejam capazes de auxiliar constantemente o Enfermeiro na acurada avaliação da pele do idoso, gestão eficaz dos riscos e tratamento cientificamente fundamentado de ambas as lesões.

\section{AGRADECIMENTOS}

Agradecemos ao CNPq, edital MCTI/CNPq no 014/2015, processo 402794/2016-8, 
pelo apoio e financiamento do macroprojeto: "Estratificação de risco e intervenções de enfermagem no diagnóstico, prevenção e tratamento de skin tears e úlcera por pressão em idosos", ao qual o presente estudo encontra-se vinculado.

\section{REFERÊNCIAS}

1. Leblanc K, Baranoski S, Holloway S, Langemo D. Validation of a New Classification System for Skin Tears. Adv. Skin Wound Care. [Internet]. 2013 [acesso em 04 out 2018]; 26(6). Disponível em: http:// dx.doi.org/10.1097/01.ASW.0000430393.04763.c7.

2. Santos LRO, Avelino FVSD, Luz MHBA, Cavalcante TB, Silva JLM, Santos CAP de S. Características demográficas e clínicas de pacientes internados em Unidades de Terapia Intensiva (UTI) com UP. Rev enferm. UFPE [Internet]. 2016 [acesso em 04 out 2018]; 10(Suppl1). Disponível em: https://periodicos. ufpe.br/revistas/revistaenfermagem/article/viewFile/10944/12250.

3. Caliri MHL, Santos VLC de G, Mandelbaum MHS, Costa IG. Classificação das Lesões Por Pressão Consenso NPUAP 2016 - Adaptada Culturalmente para o Brasil. SOBEST [Internet]. 2016 [acesso em 12 out 2018]. Disponível em: http://www.sobest.org.br/textod/35.

4. Fortes TML, Suffredini IB. Avaliação de pele em idoso: revisão da literatura. J Health Sci Inst. [Internet]. 2014 [acesso em 03 out 2018]; 32(1). Disponível em: https://www.unip.br/presencial/comunicacao/ publicacoes/ics/edicoes/2014/01 jan-mar/V32 n1 2014 p94a101.pdf.

5. Alvarez AM, Sandri JV de A. Envelhecimento da população e o comprometimento da Enfermagem. Rev. bras. enferm. [Internet]. 2018 [acesso em 14 out 2018]; 71(Suppl 2). Disponível em: http://dx.doi. org/10.1590/0034-7167-201871sup201.

6. Leblanc K, Baranski S, Christensen D, Langemo D, Edwards K, Holloway S, et al. The art of dressing selection. Adv. Skin Wound Care [Internet]. 2016 [acesso em 08 out 2018]; 29(1). Disponível em: http:// dx.doi.org/10.1097/01.ASW.0000475308.06130.df.

7. Acioli S, Kebian LVA, Faria MG de A, Ferraccioli P, Correa V de AF. Práticas de cuidado: o papel do enfermeiro na atenção básica. Rev. enferm. UERJ [Internet]. 2014 [acesso em jul 2018]; 22(5). Disponível em: http://dx.doi.org/10.12957/reuerj.2014.12338.

8. Instituto Brasileiro de Geografia e Estatística (IBGE). Estimativas de População 2017. [Internet]. 2017 [acesso em 04 dez 2017]. Disponível em: https://www.ibge.gov.br/estatisticas-novoportal/sociais/ populacao/9103-estimativas-de-populacao.html?\&t=resultados.

9. Departamento de Informática do SUS (DATASUS). Informações de Saúde. [Internet]. 2018 [acesso em 02 out 2018]. Disponível em: http://tabnet.datasus.gov.br/cgi/tabcgi.exe?cnes/cnv/prid02sc.def.

10. Polit DF, Beck CT. Fundamentos de pesquisa em enfermagem: avaliação de evidências para as práticas da enfermagem. 7. ed. Porto Alegre: Artmed; 2011.

11. Minayo MCS. O desafio do conhecimento: pesquisa qualitativa em saúde. 10. ed. São Paulo: Hucitec; 2007.

12. Rocha LES, Ruas E de FG, Santos JAD, Lima C de A, Carneiro JA, Costa FM da. Prevenção de úlceras por pressão: avaliação do conhecimento dos profissionais de enfermagem. Cogitare enferm. [Internet]. 2015 [acesso em 10 out 2018]; 20(3). Disponível em: http://dx.doi.org/10.5380/ce.v20i3.41750.

13. Vasconcelos JMB, Caliri MHL. Ações de enfermagem antes e após um protocolo de prevenção de lesões por pressão em terapia intensiva. Esc. Anna Nery [Internet]. 2017 [acesso em 02 out 2018]; 21(1). Disponível em: http://dx.doi.org/10.5935/1414-8145.20170001.

14. Ferreira SRS, Périco LAD, Dias VRFG. A complexidade do trabalho do enfermeiro na Atenção Primária à Saúde. Rev. bras. enferm. [Internet]. 2018 [acesso em 04 out 2018]; 71(Suppl1). Disponível em: http:// 
15. Paranhos WY, Santos VLCG. Avaliação de risco para úlceras de pressão por meio da Escala de Braden, na língua Portuguesa. Rev. Esc. Enf. USP [Internet]. 1999 [acesso em 08 out 2018]; 33(n.esp):191-206.

Disponível em: http://www.ee.usp.br/reeusp/upload/pdF/799.pdF.

16. Strazzieri-pulido KC, Santos VLC de G, Carville K. Cultural adaptation, content validity and inter-rater reliability of the STAR Skin tears Classification System. Rev Lat Am Enfermagem [Internet]. 2015 [acesso em 08 out 2018]; 23(1). Disponível em: http://dx.doi.org/10.1590/0104-1169.3523.2537.

17. Santos ICRV, Santos Júnior JL dos, Ribeiro LL, Xavier R de F, Almeida RB de, Morato JEM. Usabilidade do sistema de classificação de feridas por cor. Ciênc. cuid. saúde. [Internet]. 2017 [acesso em 03 out 2018]; 16(4). Disponível em: http://dx.doi.org/10.4025/cienccuidsaude.v16i4.34436.

18. Borghardt AT, Prado TN do, Araújo TM de, Rogenski NMB, Bringuente ME de O. Evaluation of the pressure ulcers risk scales with critically ill patients: a prospective cohort study. Rev. Latino-Am. Enferm. [Internet]. 2015 [acesso em 06 out 2018]; 23(1). Disponível em: http://dx.doi.org/10.1590/01041169.0144.2521.

19. Leblanc K, Baranoski S. Skin tears: best practices for care and prevention. Nursing [Internet]. 2014 [acesso em 07 out 2018]; 44(5). Disponível em: https://dx.doi.org/10.1097/01. NURSE.0000445744.86119.58.

20. Athlin AM, Engström M, Gunningberg L, Baath C. Heel pressure ulcer, prevention and predictors during the care delivery chain - when and where to take action? A descriptive and explorative study. Scand J Trauma Resusc Emerg Med [Internet]. 2016 [acesso em 07 out 2018]; 24(1). Disponível em: http:// dx.doi.org/10.1186/s13049-016-0326-0.

21. Prado YS do, Tiengo $A$, Bernardes $A C B$ e. A influência do estado nutricional no desenvolvimento de lesões por pressão em pacientes suplementados. RBONE [Internet]. 2017 [acesso em 07 out 2018]; 11(68). Disponível em: http://www.rbone.com.br/index.php/rbone/article/view/632/494.

22. Moore Z, Cowman S, Conroy RM. A randomised controlled clinical trial of repositioning, using the $30^{\circ}$ tilt, for the prevention of pressure ulcers. J. Clin. Nurs. [Internet]. 2011 [acesso em 06 out 2018]; 20(1718). Disponível em: http://dx.doi.org/10.1111/j.1365-2702.2011.03736.x.

23. Moore Z, Etten MV. Ten top tips: repositioning a patient to prevent pressure ulcers. Wounds International. [Internet]. 2014 [acesso em 06 out 2018]; 5(3). Disponível em: https://www.researchgate.net/ publication/266734622 Ten top tips repositioning a patient to prevent pressure ulcers.

24. National Pressure Ulcer Advisory Panel, European Pressure Ulcer Advisory Panel, Pan Pacific Pressure Injury Alliance. Prevention and Treatment of Pressure Ulcers: quick reference guide. [Internet] Australia: Cambridge Media; 2014 [acesso em 08 out 2018]. Disponível em: http://www.epuap.org/wp-content/ uploads/2010/10/Quick-Reference-Guide-DIGITAL-NPUAP-EPUAP-PPPIA-16Oct2014.pdf.

25. Peres GRP, Strazzieri-pulido KC, Santos VLCG. Prevenção de lesões por fricção. In: Domansky RC, Borges EL, organizadores. Manual para prevenção de lesões de pele: recomendações baseadas em evidência. 2.ed. Rio de Janeiro: Editora Rubio; 2014.

26. Garbaccio JL, Ferreira AD, Pereira ALGG. Self-skincare knowledge and practice described by elderly persons in the mid-west of Minas Gerais. Rev. Bras. Geriatr. Gerontol. [Internet]. 2016 [acesso em 07 out 2018]; 19(1). Disponível em: http://dx.doi.org/10.1590/1809-9823.2016.14237.

27. National pressure ulcer advirosy panel (NPUAP). Pressure Injury Stages. [Internet]. 2016 [acesso em 10 out 2018]. Disponível em: http://www.npuap.org/resources/educational-and-clinical-resources/npuappressure-injury-stages/.

28. Stephen-haynesJ. The Prevention and Management of Skin Tears and Lacerations. [Internet]. WHCT; 2018 [acesso em 18 dez 2019]. Disponível em: https://www.woundsinternational.com/uploads/ resources/57c1a5cc8a4771a696b4c17b9e2ae6f1.pdf. 
29. lizaka S, Kaitani T, Nakagami G, Sugama J, Sanada H. Clinical validity of the estimated energy requirement and the average protein requirement for nutritional status change and wound healing in older patients with pressure ulcers: A multicenter prospective cohort study. Geriatr Gerontol Int. [Internet]. 2014 [acesso em 03 ou 2018]; 15(11). Disponível em: http://dx.doi.org/10.1111/ggi.12420.

30. Santos El dos. Skin tear treatment and prevention by nurses: an integrative literature review. Rev. Gaúch. Enferm. [Internet]. 2014 [acesso em 03 out 2018]; 35(2). Disponível em: http://dx.doi. org/10.1590/1983-1447.2014.02.45178.

Recebido: 27/02/2019

Finalizado: 05/02/2020

Editora associada: Susanne Elero Betiolli

Autor Correspondente:

Francisco Reis Tristão

Universidade Federal de Santa Catarina

Rd. Baldicero Filomeno, 8183 - 88064002 - Florianópolis, SC, Brasil

E-mail: franciscoreistristao@hotmail.com

Contribuição dos autores:

Contribuições substanciais para a concepção ou desenho do estudo; ou a aquisição, análise ou interpretação de dados do estudo - KMZ

Aprovação da versão final do estudo a ser publicado - KSAH, CFS, SME, ASV

Responsável por todos os aspectos do estudo, assegurando as questões de precisão ou integridade de qualquer parte do estudo - FRT, JBRG 